\title{
X-ray magnetic circular dichroism study of the magnetic anisotropy on $\mathrm{TbMnO}_{3}$
}

\author{
V. Cuartero, ${ }^{1, *}$ S. Lafuerza, ${ }^{1}$ G. Subías, ${ }^{2}$ J. García, ${ }^{2}$ E. Schierle, ${ }^{3}$ J. Blasco, ${ }^{2}$ and J. Herrero-Albillos ${ }^{2,4}$ \\ ${ }^{1}$ ESRF-The European Synchrotron, 71, Avenue des Martyrs, Grenoble, France \\ ${ }^{2}$ Departamento de Física de la Materia Condensada, Instituto de Ciencia de Materiales de Aragón, \\ CSIC-Universidad de Zaragoza, C/Pedro Cerbuna 12, 50009 Zaragoza, Spain \\ ${ }^{3}$ Helmholtz Zentrum Berlin Mat \& Energie, D-12489 Berlin, Germany \\ ${ }^{4}$ Centro Universitario de la Defensa, Carretera de Huesca s/ $n$, 50090 Zaragoza, Spain
}

(Received 23 December 2014; revised manuscript received 9 March 2015; published 7 April 2015)

\begin{abstract}
The magnetic anisotropy of $\mathrm{TbMnO}_{3}$ was explored by means of polarized x-ray absorption spectroscopy and x-ray magnetic circular dichroism (XMCD) measurements at the $\mathrm{Mn} L_{2,3}$ and $\mathrm{Tb} M_{4,5}$ edges as a function of temperature and magnetic-field intensity. The selective magnetometry measurements were compared with the macroscopic magnetic properties on single crystals. XMCD measurements at the $\mathrm{Tb} M_{4,5}$ edge as a function of the magnetic field reproduces quite well the macroscopic magnetic anisotropy at low temperatures with the $\mathrm{Tb}$ moments staying confined along their Ising axis within the $a b$ plane, whereas a weak XMCD signal is observed at the $\mathrm{Mn} L_{2,3}$ edge. These results point out that $\mathrm{Tb}^{3+}$ single-ion anisotropy is the only responsible for the magnetic anisotropy on this multiferroic compound at high magnetic fields. Moreover, we found $\operatorname{Mn} L_{2,3}$ XMCD measurements show that the cycloidal antiferromagnetic order is almost unaffected by the applied magnetic field at low temperatures under an applied magnetic field. Therefore, we discuss that this strong Ising nature of $\mathrm{Tb}^{3+}$ ions, through a magnetocrystalline coupling mediated by the oxygen atoms, must play an important role in the field-induced electric polarization flop and therefore in the magnetoelectric coupling on $\mathrm{TbMnO}_{3}$.
\end{abstract}

DOI: 10.1103/PhysRevB.91.165111

PACS number(s): 75.25.-j, 75.30.Gw, 75.47.Lx, 75.85.+t

\section{INTRODUCTION}

Rare-earth- $(R$-) transition-metal (TM) oxides show a great variety of intriguing phenomena originating from the coupling between the two spin subsystems [1]. Multiferroicity and magnetoelectric coupling are among them [2,3]. However, the combination of a magnetic ordered ground state together with ferroelectricity (FE) is not trivial [4,2], and many attempts have been made in past years to explain the occurrence of these two phenomena in the same phase. In the case of type-II multiferroics [5] where FE is driven by a noncollinear magnetic ordering of the TM sublattice, the inversion symmetry breaking is due to the anisotropic exchange interaction also known as the Dzyaloshinskii-Moriya interaction [6], which promotes a net electric polarization. The electric polarization is then given by $\vec{P} \sim \lambda_{s o} \vec{r}_{i j} \times\left(\vec{S}_{i} \times \vec{S}_{j}\right)$, where $\vec{S}_{i, j}$ refers to the magnetic moment of the two first neighbors $i, j$, separated by a distance $\vec{r}_{i j}$, and $\lambda_{S O}$ is the spin-orbit coupling constant from which it is inferred that electric and magnetic properties are completely coupled.

This is the case for $\mathrm{TbMnO}_{3}$, an orthorhombic perovskite (space group Pbnm) where a sinusoidal-wave-type ordering of the $\mathrm{Mn}^{3+}$ spins, lying along $\mathbf{b}$ axis, order antiferromagnetically along the $\mathbf{c}$ axis (so-called $A_{\mathrm{y}}$-type order). This type of spin order has an onset at $T_{\mathrm{N}, \mathrm{Mn}} \approx 41 \mathrm{~K}$. Upon cooling in zero fields a second magnetic transition into a spiral phase occurs at about $28 \mathrm{~K}$, with the cycloidal spin structure oriented on the $b c$ plane and consequently the onset of spontaneous electric polarization parallel to the $\mathbf{c}$ axis. The antiferromagnetic ordering of $\mathrm{Tb}$ spins arises at $T_{\mathrm{N}, \mathrm{Tb}} \approx 7 \mathrm{~K}[7,8]$. However, several spectroscopy experiments point out that the magnetic coupling between $\mathrm{Mn}$ and $\mathrm{Tb}$, that is present even above $T_{\mathrm{N}, \mathrm{Tb}}$,

*Corresponding author: cuartero@esrf.fr plays the main role in the appearance of multiferroicity and magnetoelectric coupling [9-14]. Moreover, in an applied magnetic field a more complex behavior is found, and magnetic fields can control the direction of electric polarization [15]. Results showed a spin-flop transition of Mn moments, contained on the $b c$ plane at zero field (and $\mathbf{P} \| \mathbf{c}$ ), into a configuration lying on the $a b$ plane for $\mathbf{H} \| \mathbf{a}$ or $\mathbf{H} \| \mathbf{b}$ (then $\mathbf{P} \| \mathbf{a})$ [8]. Besides that, the macroscopic magnetic response of $\mathrm{TbMnO}_{3}$ is highly anisotropic depending on the direction of the applied external magnetic field with respect to the crystallographic axis [16], a being the easy magnetization axis and $\mathbf{c}$ the hard magnetic axis. For instance, at $9 \mathrm{~T}$ and $5 \mathrm{~K}$, the values of the magnetization are $M_{a}=6.5, M_{b}=3.7$, and $M_{c}=1.1 \mu_{\mathrm{B}} /$ f.u. (where f.u. represents formula unit), whereas $4 \mu_{\mathrm{B}}$ per ion are expected for a saturated $\mathrm{Mn}^{3+}$ lattice $\left(3 d^{4},{ }^{5} \mathrm{D}_{0}\right.$ ground state) and $9 \mu_{\mathrm{B}}$ is the theoretical magnetic moment per ion for a fully polarized $\mathrm{Tb}^{3+}$ sublattice $\left(4 f^{8},{ }^{7} \mathrm{~F}_{6}\right.$ ground state). In addition, below $7 \mathrm{~K}$, the application of an external magnetic field leads to metamagnetic transitions of the $\mathrm{Tb}^{3+}$ moments. In particular, a field parallel to the $b$ axis induces a commensurate long-range ordering of $\mathrm{Tb}$ of type $C_{x} F_{y}[17]$.

In order to disentangle the magnetic contributions responsible for the macroscopic magnetic anisotropic behavior under applied magnetic fields on $\mathrm{TbMnO}_{3}$, we have investigated the differentiated role of $\mathrm{Mn}$ and $\mathrm{Tb}$ ions by soft $\mathrm{x}$-ray absorption spectroscopy (XAS) and x-ray magnetic circular dichroism (XMCD) at $\mathrm{Tb} M_{4,5}$ and $\operatorname{Mn} L_{2,3}$ edges as a function of temperature and the intensity and direction of the applied magnetic field. We recall here that $\mathrm{TbMnO}_{3}$ is a strong insulating sample at low temperatures suffering from sample charging, which limits the applicability of the most practiced total electron yield (TEY) detection method. Therefore, we have also performed XMCD measurements in either total fluorescence yield (TFY) or reflection modes [18]. Moreover, 
as the studied samples are thick single crystals, XAS spectra measured in TFY must be corrected for self-absorption as will be addressed here. From this thorough study, it can be concluded that $\mathrm{Tb}$ magnetism dominates the observed strong magnetocrystalline anisotropy in $\mathrm{TbMnO}_{3}(\mathrm{Mn}$ contributions are at least a factor of 6 smaller). Consequently the different field-dependent behaviors of the magnetization along the three crystallographic axes reflect the Ising nature of the $\mathrm{Tb}^{3+}$ moments that is brought in by the combination of spin-orbit coupling with the low-symmetry environment of the $\mathrm{Tb}$ sites in the Pbnm structure. Moreover, this strong Ising character of $\mathrm{Tb}$ in $\mathrm{TbMnO}_{3}$ is also playing an important role in the electric polarization flop from the $\mathbf{c}$ to the $\mathbf{a}$ axis under applied magnetic fields.

\section{EXPERIMENTAL DETAILS}

$\mathrm{TbMnO}_{3}$ polycrystalline samples were prepared by the ceramic method: Stoichiometric amounts of $\mathrm{Tb}_{4} \mathrm{O}_{7}$ and $\mathrm{MnCO}_{3}$ were mixed, ground, and heated up at $1000^{\circ} \mathrm{C}$ during $12 \mathrm{~h}$ as the first step. The resulting powder was pressed into pellets and sintered at $1200^{\circ} \mathrm{C}$ during $24 \mathrm{~h}$ in air. As the last step, the samples were repressed into pellets and sintered at $1400^{\circ} \mathrm{C}$ under $\mathrm{Ar}$ atmosphere during $48 \mathrm{~h}$. X-ray diffraction patterns were collected with a Rigaku D/max-B diffractometer with a copper rotating anode and a graphite monochromator in order to select the $\mathrm{Cu} K \alpha$ wavelength $(\lambda=1.5418 \AA)$. The patterns were in agreement with a single phase orthorhombic perovskite, space group Pbnm, and the samples were then used as the feed and seed rods for the single-crystal growth. $\mathrm{TbMnO}_{3}$ single crystals were grown by the floating-zone method using a homemade furnace with two semielliptical mirrors [19]. The feed and seed rods were rotating in opposite directions at $\pm 20 \mathrm{rpm}$, and the growth speed was $8 \mathrm{~mm} / \mathrm{h}$. The growth was carried out under an Ar atmosphere, and a boule of $7 \mathrm{~cm}$ was obtained. Three crystals were cut so that their faces were perpendicular to the $\mathbf{a}, \mathbf{b}$, and $\mathbf{c}$ crystallographic axes.

The macroscopic magnetization measurements as a function of temperature and applied magnetic field up to $60 \mathrm{kOe}$ were performed with a commercial physical property measurement system (PPMS) from Quantum Design using the vibrating sample magnetometry configuration [17].

Soft x-ray spectroscopy measurements were performed at the high-field diffractometer of the UE46-PGM1 (where PGM represents a plane grating monochromator) beamline at the BESSY-II synchrotron (Helmholtz-Zentrum, Berlin, Germany). XAS and XMCD measurements at the $\mathrm{Tb} M_{4,5}$ edges (from 1220 to $1290 \mathrm{eV}$ ) and the $\mathrm{Mn} L_{2,3}$ edges (from 635 to $665 \mathrm{eV}$ ) were carried out from $100 \mathrm{~K}$ down to $5 \mathrm{~K}$ with an applied magnetic field up to $6 \mathrm{~T}$ oriented along the three different crystallographic axes. XMCD spectra were obtained as the difference between two absorption spectra measured with opposite x-ray beam helicity (right $\mu^{+}$and left $\mu^{-}$circular polarizations, $\mathrm{XMCD}=\mu^{+}-\mu^{-}$) and a fixed magnetic-field direction. The dichroic signals are normalized to the maximum intensity of the averaged XAS spectrum obtained by merging $\left(\mu^{+}+\mu^{-}\right) / 2$ and will be represented as a percentage of the XAS signal. The helicity of the $\mathrm{x}$-ray beam was changed by reversing the phase of the helical undulator, and the degree of circular polarization was $90 \%$. (a) Reflection geometry

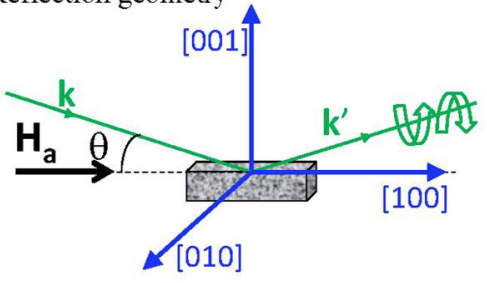

(b) TFY detection

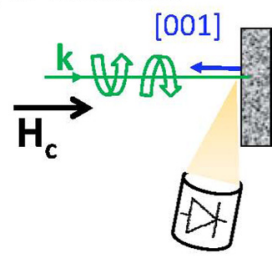

(c) TEY detection

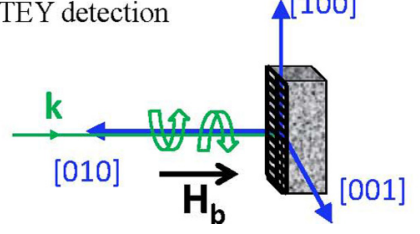

FIG. 1. (Color online) Configurations of the detection modes used in the experiment. (a) Reflection geometry where there is a small angle $\left(\leqslant 8^{\circ}\right)$ between the direction of the magnetic field and the photon's propagation vector. (b) TFY where the fluorescence emitted photons are detected by a photodiode placed at $\sim 80^{\circ}$ with respect to the incoming photons to reduce Compton scattering. (c) TEY where the electron-scattering cascade following the Auger decay is measured via the sample drain current.

A PGM was used, and the primary slits were opened in order to find the best compromise between photon flux and energy resolution $(1.2 \mathrm{eV})$.

Three detection modes were used to carry out the spectroscopic measurements at the $\mathrm{Tb} M_{4,5}$ edges under ultra-highvacuum conditions: TFY, reflection, and TEY. Here reflectivity and TFY measurements will be presented, more sensitive to the bulk properties of the system. The corresponding geometrical configurations are plotted in Fig. 1. XMCD measurements in reflection geometry were performed in a cleaved single crystal with the [001] surface normal in order to get a clean surface [Fig. 1(a)]. $X$ rays impinged the sample at a fixed grazing incidence angle of $\theta \sim 6^{\circ}-8^{\circ}$, and the magnetic field was applied for either $\mathbf{H} \| \mathbf{a}$ or $\mathbf{H} \| \mathbf{b}$ allowing magnetization of the sample parallel to its surface. The outgoing photons were collected by a photodiode. Simultaneous TFY and TEY detection modes were used for the $\mathbf{H} \| \mathbf{c}$ configuration in a polished single crystal with the [001] surface normal covered with a graphite mesh to ensure electrical conductivity at low temperatures. A photodiode was placed at about $80^{\circ}$ with respect to the incident beam as can be seen in Fig. 1(b). The self-absorption effects and their correction will be discussed in the next section. At the $\operatorname{Mn} L_{2,3}$ edges, only TEY detection was used on a polished single crystal with the [010] surface normal covered with a graphite mesh [Fig. 1(c)].

\section{RESULTS AND DISCUSSION}

\section{A. XMCD at the Tb $M_{4,5}$ edge}

The $\mathrm{Tb} M_{4,5}$-edge $\mu^{+}$and $\mu^{-}$spectra measured in reflection geometry with an applied magnetic field $\mathbf{H} \| \mathbf{a}$ of $5 \mathrm{~T}$ at $5 \mathrm{~K}$ are shown in Fig. 2 as well as the XMCD signals with the applied magnetic field parallel to both [100] and [010] crystallographic directions, following the experimental setup described in Fig. 1(a). The Tb $3 d-4 f$ absorption spectrum 


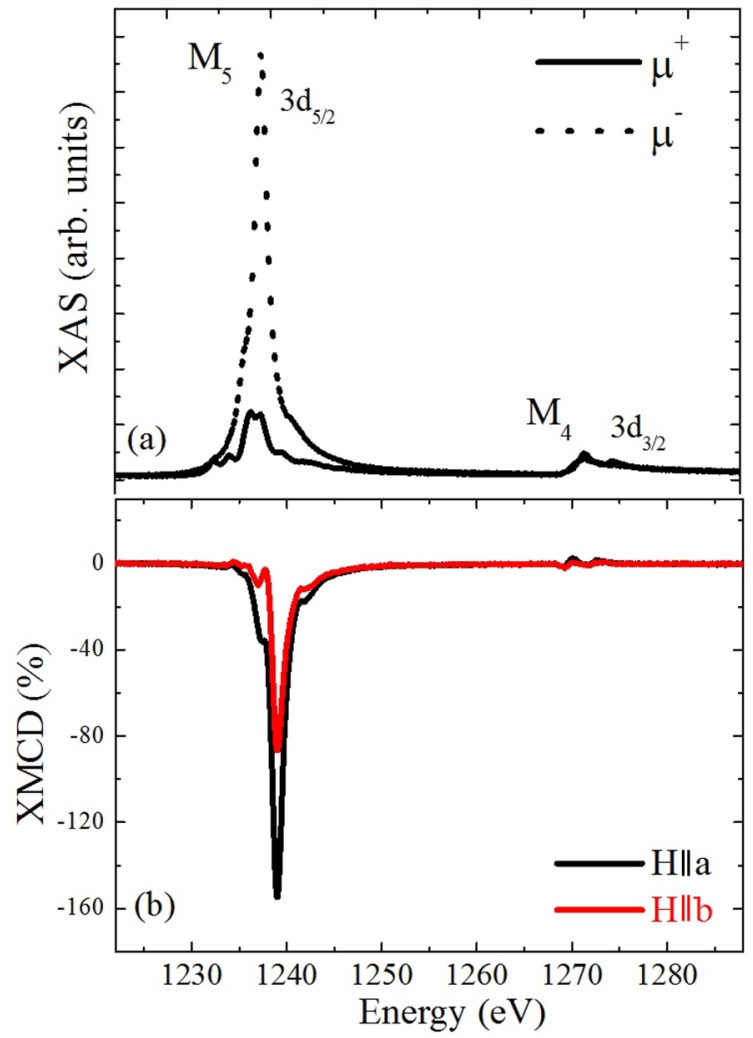

FIG. 2. (Color online) (a) XAS spectra taken at the $M_{4,5}$ edges of Tb with right $\left(\mu^{+}\right)$and left $\left(\mu^{-}\right)$circular polarizations for the $\mathbf{H} \| \mathbf{a}$ configuration at $T=5 \mathrm{~K}$ and $H=5 \mathrm{~T}$. (b) Normalized XMCD spectra with a 5-T magnetic field applied along the [100] and [010] crystallographic directions at $T=5 \mathrm{~K}$.

is characterized by two structures $\left(M_{5}\right.$ and $\left.M_{4}\right)$ well separated in energy by the strong spin-orbit interaction. The dichroism is very strong as expected for a rare-earth atom having a high spin magnetic moment. The high XMCD intensity at the $M_{5}$ edge compared to the low intensity at the $M_{4}$ edge indicates a strong contribution from the orbital moment of the Tb ion [20].

The overall spectral line shape of the XMCD signal is very similar for the two crystallographic directions and resembles other XMCD spectra measured in different terbium systems at the $\mathrm{Tb} M_{4,5}$ edge [21-23]. However, the amplitude of the dichroism is considerably different: $160 \%(\mathbf{H} \| \mathbf{a})$ and $85 \%(\mathbf{H} \| \mathbf{b})$.

For the $\mathbf{H} \| \mathbf{c}$ configuration, we used TFY detection as described in Fig. 1(b). Under this geometry, self-absorption effects become important due to the longer penetration depth of $\mathrm{x}$ rays. The self-absorption effects can be corrected by taking into account geometrical factors in such a way that the experimental fluorescence absorption spectra $\mu_{F}$ results into [24]

$$
\mu_{F}(E)=\frac{\left(\frac{\Omega}{4 \pi}\right) \varepsilon_{f} \mu(E)}{\mu_{T}(E)+\mu_{T}\left(E_{F}\right) \frac{\sin \theta}{\sin \varphi}},
$$

$\mu_{T}(E)$ being the total absorption coefficient including contributions from the edge of interest and from other edges of all atoms in the compound $\left[\mu(E)+\mu_{\mathrm{bkg}}(E)\right]$ and $\mu_{T}\left(E_{F}\right)$ being the total absorption coefficient at the energy of the

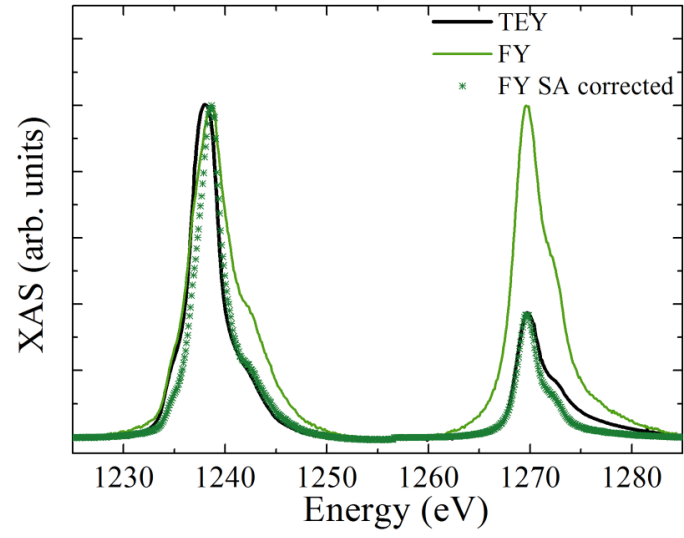

FIG. 3. (Color online) XAS spectra normalized to the maximum of the $M_{5}$ edge measured in TEY, in FY, and self-absorption (SA) corrected (points) at $5 \mathrm{~K}$ without an applied magnetic field.

fluorescence photons $E_{F} . \theta$ and $\phi$ are the angles between the sample surface and the direction of the incoming and the detected fluorescence photons, respectively. Finally, $\Omega / 4 \pi$ is the solid angle covered by the detector, and $\varepsilon_{f}$ is the fluorescence yield of the absorber atom.

In this case, $\mu_{T}(E)$ can be approximated to $\mu_{T}(E)=$ $\mu(E)+k$, where $k$ is constant since the energy range at the $\operatorname{Tb} M_{4,5}$ edges is small. Taking into account Eq. (1) and grouping all the constant factors, we can rewrite the absorption coefficient without self-absorption effects as follows:

$$
\mu(E)=\frac{C_{1} \mu_{F}(E)}{C_{2}-\mu_{F}(E)}=\mu_{T E Y}(E)-\mu_{\mathrm{bkg}}(E)
$$

Therefore, TEY-detected $\mu^{+}$and $\mu^{-}$experimental spectra (background subtracted) were fitted to the corresponding $\mu_{F}(E)$ measurement to obtain $C_{1}$ and $C_{2}$ fitting constants. To do so, TEY and TFY spectra were measured simultaneously. Then, Eq. (2) was applied to TFY spectra to finally obtain the absorption coefficient $\mu(E)$ free of self-absorption effects. The estimated error of this procedure is about $10 \%$, which is inside the total estimated experimental uncertainty. The comparison among $\mu_{T E Y}, \mu_{F}$, and the final $\mu$ obtained after the self-absorption correction can be seen in Fig. 3. The ratio between $M_{5}$ and $M_{4}$ edges is satisfactorily corrected, and the line shape changes accordingly.

After the self-absorption correction, the normalized $\mathrm{Tb} M_{4,5}$-edge XAS and XMCD spectra at $5 \mathrm{~K}$ with the magnetic field of $5 \mathrm{~T}$ applied along the crystallographic $c$ axis are shown in Fig. 4. The amplitude of the dichroic signal is $28 \%$, much smaller than for $\mathbf{H} \| \mathbf{a}$ and $\mathbf{H} \| \mathbf{b}$ configurations. We note here that the maximum values of the XMCD signals along the three crystallographic directions are strongly anisotropic and proportional to the magnetizations $M_{a}, M_{b}$, and $M_{c}$, respectively, at $T=5 \mathrm{~K}$ and $H=5 \mathrm{~T}$ [16].

To track the evolution of the $\mathrm{Tb}$ contribution to the magnetization anisotropy, we measured the $\mathrm{Tb} M_{4,5}$-edge XMCD signals as a function of magnetic field at $T=5 \mathrm{~K}$, below $T_{\mathrm{N}, \mathrm{Tb}}$. Figure 5 shows the integrated XMCD signal at the $\mathrm{Tb} M_{5}$ edge normalized by the maximum of the corresponding averaged XAS spectrum for the complete set of values of the applied magnetic field $(0-5 \mathrm{~T})$ along the three 


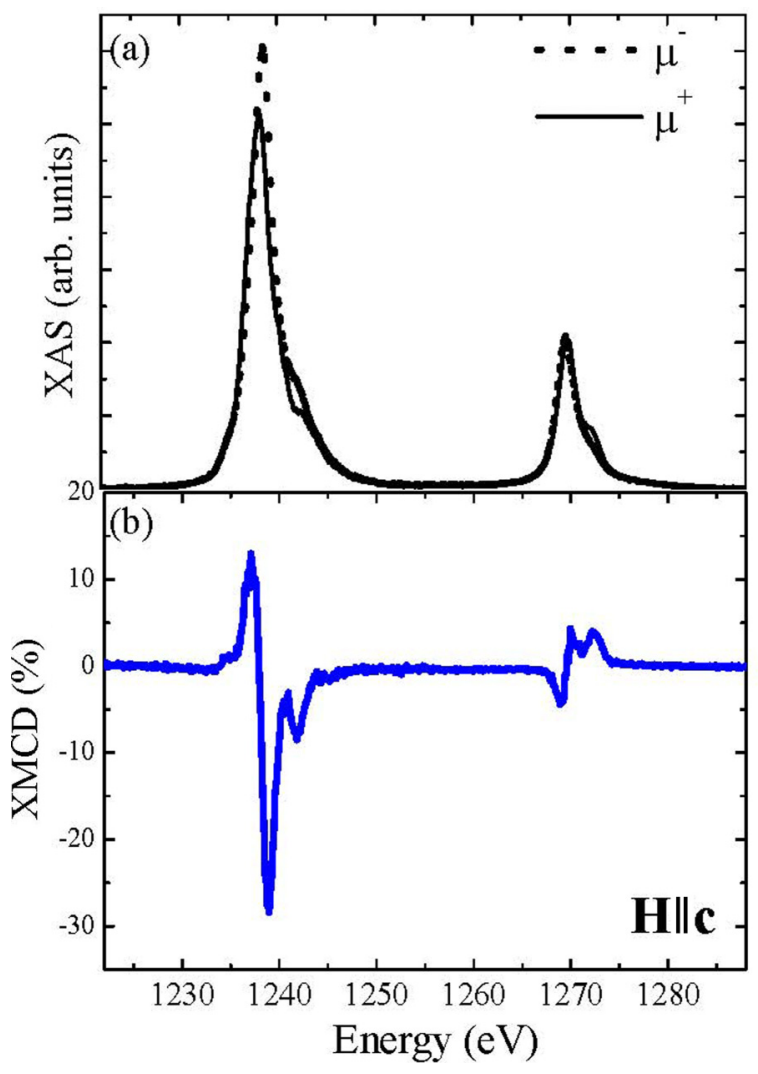

FIG. 4. (Color online) (a) Self-absorption corrected XAS spectra taken at the $M_{4,5}$ edges of Tb with right $\left(\mu^{+}\right)$and left $\left(\mu^{-}\right)$circular polarizations for the $\mathbf{H} \| \mathbf{c}$ configuration at $T=5 \mathrm{~K}$ and $H=5 \mathrm{~T}$. (b) Normalized XMCD spectra with a 5-T magnetic field applied along the [001] crystallographic direction at $T=5 \mathrm{~K}$.

crystallographic a, b, and $\mathbf{c}$ axes. In Fig. 5 the field dependence macroscopic magnetization measurements at $T=5 \mathrm{~K}$ are also shown, which indicate a strong anisotropy with the local moments almost confined on the $a b$ plane but also show anisotropic magnetization within this $a b$ plane. Indeed, the change in the evolution of the magnetization slope around $4.5 \mathrm{~T}$ along the $\mathbf{b}$ axis is well reproduced by the XMCD integrated

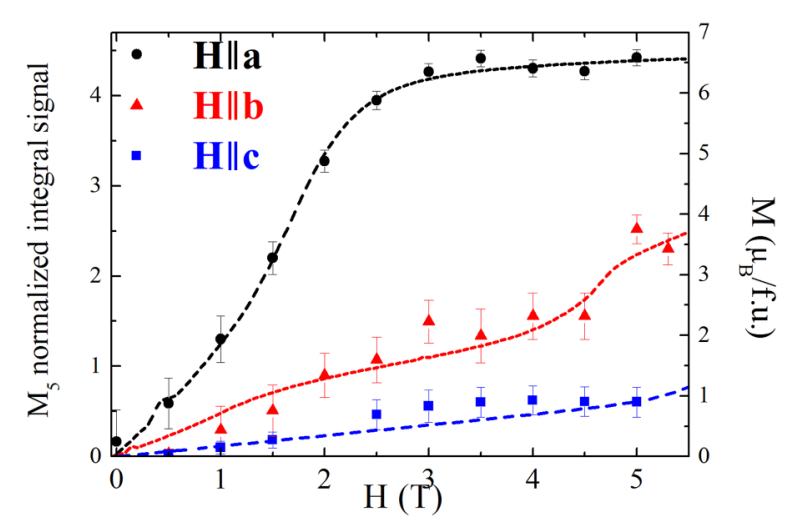

FIG. 5. (Color online) Macroscopic magnetization (dotted lines) as a function of the external field (taken at $T=5 \mathrm{~K}$ ) along the three crystallographic axes of the $\mathrm{TbMnO}_{3}$ single crystal compared to the normalized $\mathrm{Tb} M_{5}$ edge integrated XMCD signal (solid symbols). signal within the error bars. At $5 \mathrm{~K}$, Tb moments exhibit an incommensurate antiferromagnetic order at zero fields, so this step in the slope for $\mathbf{H} \| \mathbf{b}$ at 4.5-5 $\mathrm{T}$ corresponds well with the metamagnetic transition observed in the results previously reported [16,17]. In addition, the saturation trend along the $\mathbf{a}$ axis above $3 \mathrm{~T}$ is also followed by the integrated signal obtained for $\mathbf{H} \| \mathbf{a}$. For $\mathbf{H} \| \mathbf{c}$, magnetization increases almost linearly with increasing field, whereas staying far smaller than $M_{a b}$. This field behavior is also well reproduced by the XMCD integrated signal obtained for $\mathbf{H} \| \mathbf{c}$. Then, the strong magnetic anisotropy in $\mathrm{TbMnO}_{3}$ is mostly related to the Tb-sublattice magnetism with $\mathrm{Tb}$ magnetic moments mainly contained within the $a b$ plane.

Aiming at elucidating the underlying physics the experimental spectra were fitted with theoretical ones. The calculations of absorption and dichroic spectra were based on atomic multiplets performed with the CTM4XAS program [25]. In order to simulate the Tb XAS spectra, we considered the $\mathrm{Tb}^{3+}$ single ion for initial $3 d^{10} 4 f^{8}$ and final $3 d^{9} 4 f^{9}$ states, and we included the crystal-field point symmetry by branching from $\mathrm{SO}_{3}$ symmetry to $O_{h}$, then lowering the symmetry to $D_{4 h}$, and finally to $C_{4 h}$, according to the Butler branching rules for the symmetry elements [26]. The relevant atomic Hartree-Fock values of the electrostatic integrals $\left(F^{\mathrm{k}}\right)$ and exchange parameters $\left(G^{\mathrm{k}}\right)$ calculated with Cowan's code [27] were scaled down to $80 \%$ and $99 \%$, respectively. The comparison between the experimental spectra obtained from the merge of the XAS spectra along the three crystallographic directions and the theoretical (isotropic average) calculation is shown in Fig. 6. Both XAS spectra are normalized to the maximum of the $M_{5}$ edge.

The agreement of the calculations with the experimental data is reasonable and reproduces the main features of the spectra. Minor differences are found in the line shape and can be attributed to the fact that in reflection geometry, the shape of the XAS and XMCD spectra depends not only on absorption, but also on dispersion. However, the intensity of the experimental XMCD signal is reduced with respect to the atomic multiplet calculation. To determine the value of the $\mathrm{Tb}$ magnetic moment, we exploited the calculated spectra to check first the validity of the application of the sum rules [28,29] to this system. In their application we followed Ref. [28], considering that the dipolar transition rule for $\mathrm{Tb}^{3+}\left(4 f^{8}\right)$ at $M_{4,5}$ edges gives $c=2$ ( $d$ initial state) and $l=3$ ( $f$ final state). Then, the expectation values of the projection of the orbital and spin moments are as follows:

$$
\left\langle L_{z}\right\rangle=\frac{q(14-n)}{r}, \quad\left\langle S_{z}\right\rangle=\frac{(5 p-3 q)(14-n)}{4 r}-3\left\langle T_{z}\right\rangle,
$$

where $q=\int_{M_{4}+M_{5}} d w\left(\mu^{+}-\mu^{-}\right), \quad p=\int_{M_{5}} d w\left(\mu^{+}-\mu^{-}\right)$, and $r=\int_{M_{4}+M_{5}} d w\left(\mu^{+}+\mu^{-}\right) / 2$, whereas $\left\langle T_{z}\right\rangle$ is the expectation value of the intra-atomic magnetic dipole operator. Here for the $\mathrm{Tb}^{3+}$ ion the atomic value $T_{z} / S_{z}=-0.081$ is considered as it has been previously calculated [30]. The $L_{z}$ and $S_{z}$ values derived from the sum rules applied to the calculated spectra are -3.3 and -2.6 , respectively, being the $\mathrm{Tb}$ total magnetic moment $m_{z}=-\left(2 S_{z}+L_{z}\right)=8.6 \pm 0.2 \mu_{\mathrm{B}}$. These values agree within $10 \%$ with the expected ones for a free $\mathrm{Tb}^{3+}\left(4 f^{8}\right)$ with a Hund's rule ground state $J=6$, namely, 


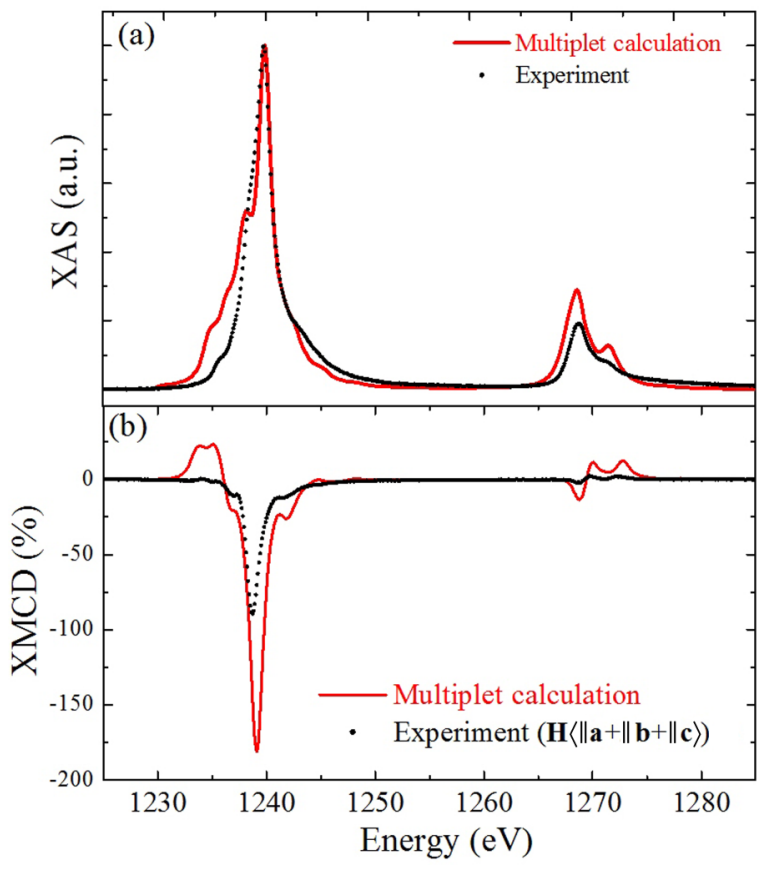

FIG. 6. (Color online) Comparison of experimental (dots) (a) average XAS spectra and (b) average XMCD spectra merged along the three crystallographic directions $(\mathbf{H}\langle\|\mathbf{a}+\| \mathbf{b}+\| \mathbf{c}\rangle)$ taken on $\mathrm{TbMnO}_{3}$ cleaved single crystals with the results of multiplet calculations (solid lines). For the experiment, temperature and external magnetic field were $5 \mathrm{~K}$ and $5 \mathrm{~T}$, respectively.

$L=3, S=3$, and a theoretical saturation moment of $9 \mu_{\mathrm{B}}$. We then applied the sum rules to the experimental spectra merged along the three crystallographic directions $(\langle\mathbf{a}+\mathbf{b}+\mathbf{c}\rangle)$ under an applied magnetic field of $5 \mathrm{~T}$. The expectation values for $L_{z}$ and $S_{z}$ are -1.6 and -2.5 , respectively, giving a Tb magnetic moment of $5.8 \mu_{\mathrm{B}}$. The experimental $\mathrm{Tb}$ magnetic moment found at $5 \mathrm{~T}$ is much smaller than the single-ion saturation value, but it is close to the merged value of the magnetic moment along the $\mathbf{a}$ and $\mathbf{b}$ directions $\left(5.6 \mu_{\mathrm{B}}\right)$, which corresponds to the plane where $\mathrm{Tb}$ magnetic moments are mostly confined.

This magnetic behavior is in agreement with the strong Ising character of $\mathrm{Tb}^{3+}$ and the four-sublattice model proposed for the antiferromagnetic state below $T_{\mathrm{N}, \mathrm{Tb}}$ in various perovskite compounds [31,32]. $\mathrm{Tb}^{3+}\left(4 f^{8}\right)$ is a non-Kramers' ion being ${ }^{7} \mathrm{~F}_{6}$ the lowest-lying free-ion term, but in $\mathrm{TbMnO}_{3}$, similar to $\mathrm{TbAlO}_{3}$ [33], the crystal field of the Tb Wyckoff position $4 c$ with point symmetry $C_{s}$ (subgroup of $C_{4 h}$ ) splits this ground-state multiplet into $2 J+1=13$ singlets. As expected from the energy balance between the crystal-field splitting (tenths of meV) and the Zeeman energy splitting at $5 \mathrm{~T}(\mathrm{meV})$, the lowest crystal-field level is a quasidoublet that can be approximated by a linear combination of "pure" $|6, \pm 6\rangle$ states, corresponding to the magnetic moment of a free- $\mathrm{Tb}^{3+}$ ion, i.e., $9 \mu_{\mathrm{B}}$ [33]. Besides, the large crystalline anisotropy of $\mathrm{Tb}^{3+}$ fixes the magnetic moments of the two inequivalent $\mathrm{Tb}$ sites in Pbnm, related by a mirror reflection on the $a c$ plane, along their respective Ising axes (the anisotropy axis), resulting in noncollinear ordered arrangements within the $a b$ plane $[31,32]$. On the other hand, at zero external fields and below

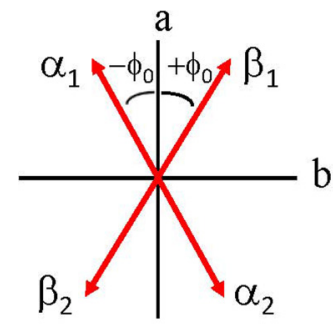

(a) $\mathrm{H}=0 \mathrm{~T}$

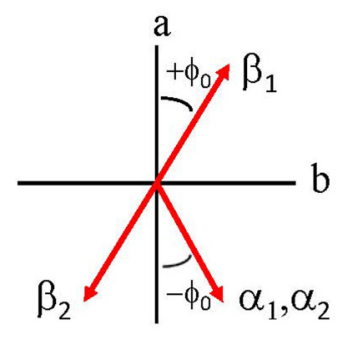

(c) $2 \mathrm{~T}<\mathrm{H}_{\mathrm{b}}<5 \mathrm{~T}$

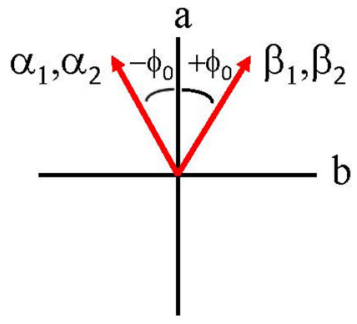

(b) $\mathrm{H}_{\mathrm{a}}>2 \mathrm{~T}$

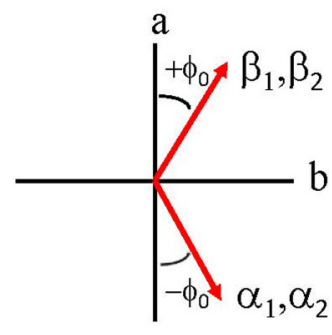

(d) $\mathrm{H}_{\mathrm{b}}>5 \mathrm{~T}$
FIG. 7. (Color online) Tb magnetic moment configuration projected on the $a b$ plane in $\mathrm{TbMnO}_{3}$ at low temperatures $(T=5 \mathrm{~K})$. (a) $H=0$, (b) $H>2.5 \mathrm{~T}$ applied along the $a$ axis, (c) $2 \mathrm{~T}<H<5 \mathrm{~T}$ applied along the $b$ axis, and (d) $H>5 \mathrm{~T}$ applied along the $b$ axis.

$T_{\mathrm{N}, \mathrm{Tb}}$ the $\mathrm{Tb}$ sublattice shows an incommensurate antiferromagnetic order [8], whereas for $T_{\mathrm{N}, \mathrm{Tb}}<T<T_{\mathrm{FE}}=27 \mathrm{~K}$ both $\mathrm{Mn}$ and $\mathrm{Tb}$ orderings have been shown to be coupled with the same wave vector [9-14], and for $T>T_{\mathrm{FE}}=27 \mathrm{~K}, \mathrm{~Tb}$ is in the paramagnetic state. Therefore, in zero field and $T>T_{\mathrm{FE}}$, the magnetic moments $\alpha_{1}, \alpha_{2}$ and $\beta_{1}, \beta_{2}$ of the four Tb sites in the orthorhombic cell are disordered along the two Ising directions $-\phi_{0}$ and $+\phi_{0}$, respectively, from the a axis with no net magnetization [Fig. 7(a)]. The application of a magnetic field parallel to the $\mathbf{a}$ or $\mathbf{b}$ axis does not align the $\mathrm{Tb}$ paramagnetic moments in the field direction but along the Ising axes of the two inequivalent $\mathrm{Tb}$ sites, resulting into pairs of aligned magnetic moments at an angle $\phi_{0}$ from the a axis [Fig. 7(b)] or the b axis [Fig. 7(d)]. However, below $T_{\mathrm{N}, \mathrm{Tb}}$ the orientation of the $\mathrm{Tb}$ moments following the applied field competes with the antiferromagnetic order, and consequently metamagnetic changes are manifested as a step behavior in the $M(H)$ curves. Then, at $T<T_{\mathrm{N}, \mathrm{Tb}}$ when $\mathbf{H} \| \mathbf{a}$, the flipping of the magnetic moments occurs at low fields about $1.5 \mathrm{~T}$, whereas if $\mathbf{H} \| \mathbf{b}$, two discontinuities are observed, and a canted antiferromagnetic order is likely to be stable between 2 and $4.5 \mathrm{~T}$. At a first approximation, we can consider the simple two-step model for the flipping of $\mathrm{Tb}$ moments along $b$ as for related perovskites. At $\mathbf{H}_{\mathbf{b}} \sim 2 \mathrm{~T}$, the magnetic moment of just one of the two inequivalent $\mathrm{Tb}$ sites is aligned along its Ising axis in the field direction [Fig. 7(c)] whereas the second one is aligned at $\mathbf{H}_{\mathbf{b}} \sim 4.5 \mathrm{~T}[$ Fig. $7(\mathrm{~d})]$ [34]. Finally, we would like to note that the magnitude of the $\mathrm{Tb}$ magnetic moment and the canted angle $\phi_{0}$ deduced from the experimental magnetization values, along the $\mathbf{a}$ and $\mathbf{b}$ axes in high magnetic fields $(>5 \mathrm{~T})$ and below $T_{\mathrm{N}}(\mathrm{Tb})$, are $8.2 \mu_{\mathrm{B}}$ 


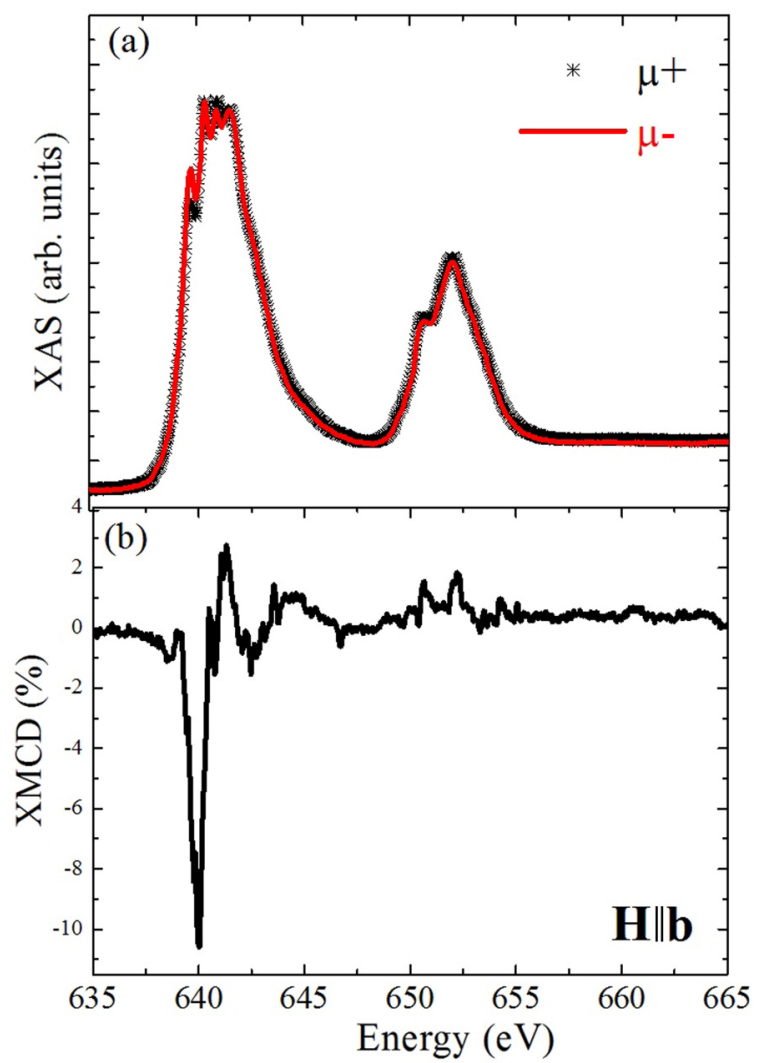

FIG. 8. (Color online) (a) XAS spectra taken at the $L_{2,3}$ edge of Mn with right $\left(\mu^{+}\right)$and left $\left(\mu^{-}\right)$circular polarizations for $\mathbf{H} \| \mathbf{b}$ configuration at $T=5 \mathrm{~K}$ and $H=5 \mathrm{~T}$. (b) Normalized XMCD spectra with a 5-T magnetic field applied along the crystallographic b axis at $T=5 \mathrm{~K}$.

and $34^{\circ}$, respectively [17], which justifies that the ground-state quasidoublet $(|6,6\rangle \pm|6,-6\rangle / \sqrt{2})$ does not couple with higher crystal field levels at low temperatures.

\section{B. XMCD at the $M n L_{2,3}$ edge}

Attention is now paid to the Mn magnetic contribution. Figure 8 shows the XMCD signal using TEY as the detection mode for $\mathbf{H} \| \mathbf{b}$ as well as XAS spectra taken at the $\operatorname{Mn} L_{2,3}$ edges with both helicities for the $\mathrm{TbMnO}_{3}$ single crystal with the [010] surface normal. Temperature and magnetic field were $5 \mathrm{~K}$ and $5 \mathrm{~T}$, respectively.

The XMCD spectrum at the $\mathrm{Mn} L_{2,3}$ edges shows a negative signal at the $L_{3}$ edge and a very weak positive signal at the $L_{2}$ edge, which indicates that the Mn sublattice is polarized by the magnetic field at low temperatures and a small net magnetic moment contribution is induced within the proposed antiferromagnetic cycloidal configuration of the Mn moments on the $a b$ plane [8]. The magnitude of the XMCD signal is much smaller (at least a factor of 8 ) than that reported for the $\mathrm{Tb} M_{4,5}$ edges (see Fig. 2) and far from the expected ratio between the theoretical magnetic moments for fully saturated $\mathrm{Mn}^{3+}$ and $\mathrm{Tb}^{3+}$ sublattices. These results indicate that the small net magnetic moment of the $\mathrm{Mn}$ atoms along the applied field direction arises from a small canting of the antiferromagnetic Mn moments (cycloidal order). Moreover,

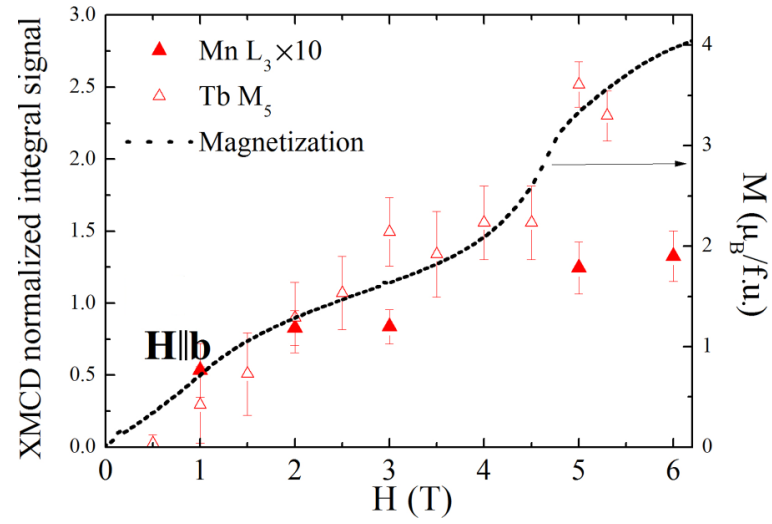

FIG. 9. (Color online) Normalized integrated XMCD intensity as a function of the external field (taken at $T=5 \mathrm{~K}$ ) along the crystallographic $b$ axis of the $\mathrm{TbMnO}_{3}$ single crystal. Solid and open triangles refer to the $\mathrm{Mn} L_{3}$, the $\mathrm{Tb} M_{5}$ integrated, and the normalized dichroic signals, respectively. The $\mathrm{Mn} L_{3}$ integrated signal is multiplied by 10 to be better visualized. The dashed line displays the reported magnetization measurement (right $y$ axis).

the signs of the Mn and Tb XMCD signals are in agreement with a parallel alignment of the $\mathrm{Mn}$ and $\mathrm{Tb}$ sublattices and parallel to the applied magnetic field, similar to other manganites [35].

The field dependence of the $\mathrm{Mn} L_{3}$ edge integrated XMCD signal normalized to the maximum of the corresponding averaged absorption spectra for $\mathbf{H} \| \mathbf{b}$ at $T=5 \mathrm{~K}$ is shown in Fig. 9. The $\mathrm{Mn} L_{3}$ edge magnetometry curve increases continuously with increasing the magnetic field, but the magnetic evolution does not follow the field dependence of the $\mathrm{Tb}$ moments. Therefore, the different field dependences of the $\mathrm{Mn}$ and $\mathrm{Tb}$ sublattice magnetizations point towards the decoupling between $\mathrm{Tb}^{3+}$ and $\mathrm{Mn}^{3+}$ net moments under applied magnetic fields in agreement with neutron-diffraction measurements that report on a $\mathrm{Tb}$ ferromagnetic ordering under applied magnetic fields $(k=0)$ whereas Mn cycloidal antiferromagnetic ordering remains unaltered $[10,17]$.

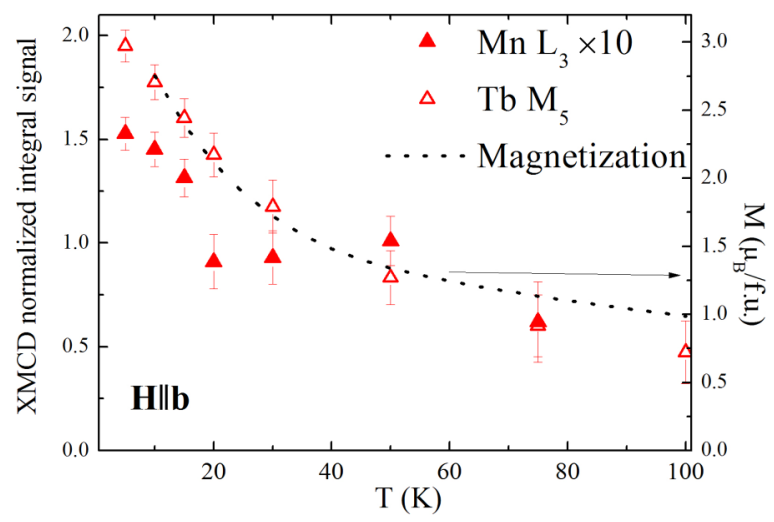

FIG. 10. (Color online) Macroscopic magnetization under a 5-T magnetic field along the $\mathbf{b}$ axis as a function of temperature (dotted line) compared with the Tb XMCD $M_{5}$ (open triangles) and $\mathrm{Mn}$ XMCD $L_{3}$ (solid triangles) integrated signals (multiplied by 10 for the sake of comparison) normalized to the maximum of their respective averaged absorption signals. 
Finally, the temperature dependence of both the Mn and the $\mathrm{Tb}$ sublattice magnetizations was measured at $H=5 \mathrm{~T}$ in the temperature range from $5 \mathrm{~K}$ up to $100 \mathrm{~K}$ for this $\mathbf{H} \| \mathbf{b}$ configuration using TEY detection as shown in Fig. 10, compared to the magnetization $M_{b}$ [intermediate behavior between the easy (a) and the hard (c) magnetization axes]. The Tb $M_{5}$ integrated XMCD signal follows the same evolution with temperature as the macroscopic magnetization, indicating the main contribution of $\mathrm{Tb}$ magnetic moments to the bulk magnetization up to temperature values well above their ordering temperature $T_{\mathrm{N}, \mathrm{Tb}}$. The $\mathrm{Mn} L_{3}$ integrated XMCD signal also shows a gradual decrease with increasing the temperature, which can be ascribed to the decreasing of the net magnetic moments of the Mn atoms on heating.

\section{CONCLUSIONS}

The separated role of $\mathrm{Tb}$ and $\mathrm{Mn}$ sublattices on the magnetic properties of $\mathrm{TbMnO}_{3}$ under applied magnetic fields has been studied by spectroscopic techniques sensitive to $f$ and $d$ states, respectively. At zero field, $\mathrm{Tb}$ and $\mathrm{Mn}$ magnetic orderings are coupled below $T_{\mathrm{FE}}=28 \mathrm{~K}$ [14] and are still correlated below $T_{\mathrm{N}}^{\mathrm{Tb}}[9,13]$, different from other manganites where $R=\mathrm{Dy}, \mathrm{Ho}$ [34]. Here, reflectivity and TFY measurements of XMCD have been performed on $\mathrm{TbMnO}_{3}$ and show that the amplitude of the dichroic signals at $\mathrm{Tb} M_{4,5}$ edges reproduce the macroscopic magnetic anisotropy of $\mathrm{TbMnO}_{3}$ as a function of the magnetic field at low temperatures $(5 \mathrm{~K})$. These results univocally demonstrate that the $\mathrm{Tb}$ sublattice is the only responsible for the magnetic anisotropy in this compound. In addition, $\mathrm{Tb}$ magnetization never reaches saturation, so $\mathrm{Tb}$ is not simply magnetized along the field direction. This magnetic behavior is perfectly explained by considering the strong Ising character of the $\mathrm{Tb}^{3+}$ ions $(J=6)$ and the low local symmetry of the nonequivalent $\mathrm{Tb}$ sites in the Pbnm structure. Therefore, $\mathrm{Tb}$ moments stay along their Ising axes within the $a b$ plane. By contrast, the Mn sublattice shows weak XMCD signals as a result of a small projection along the direction of the applied magnetic field along the $\mathbf{b}$ axis. Besides that, the small Mn moment has been found to be parallel to the $\mathrm{Tb}$ moment, which confirms that Mn moments are canted following the applied field direction. This result proves the decoupling between the $\mathrm{Tb}$ and the Mn magnetic sublattices by the application of an external magnetic field in agreement with previous neutron-diffraction measurements [17], which lead to a noncollinear arrangement of the type $C_{x} F_{y}$ (Bertaut's notation [36]) of the $\mathrm{Tb}$ moments together with a cycloid magnetic structure of the Mn moments on the $a b$ plane when at least $5 \mathrm{~T}$ are applied along the $\mathbf{b}$ direction.

Finally and according to the differentiated $\mathrm{Tb}$ and $\mathrm{Mn}$ sublattice contributions to the magnetic response in $\mathrm{TbMnO}_{3}$ under applied magnetic fields, shown by this experimental paper, we discuss the role of the $\mathrm{Tb}^{3+}$ single-ion anisotropy in the macroscopic magnetoelectrical response of this system. The observed strong crystalline anisotropy of $\mathrm{Tb}^{3+}$ forces $\mathrm{Tb}$ moments to be mostly confined along their Ising axes on the $a b$ plane, and only when applying $H$ along those axes, is the electric polarization observed to be flopped from the c to the $\mathbf{a}$ axis for both $\mathbf{H} \| \mathbf{a}$ and $\mathbf{H} \| \mathbf{b}$ configurations. In both cases the new $P$ orientation $\left(P_{\mathrm{a}}\right)$ is parallel to the Tb largest induced moment $\left(m_{\mathrm{a}}\right)$ component at high fields. Differently, the application of the magnetic field in the $\mathbf{c}$ direction does not induce any alignment of the $\mathrm{Tb}$ moments, and thus the electrical polarization remains unaltered [16]. On the other hand, the oriented Mn magnetic moment along the field direction is very small, so the cycloidal antiferromagnetic ordering of the Mn moments is poorly affected by an external magnetic field (Fig. 9) [17]. Moreover, the magnetic coupling between Mn and $\mathrm{Tb}$ sublattices under applied magnetic fields is shown to be very subtle; consequently high fields are needed to flop one of the components of the Mn cycloid (and then the $P$ direction). Therefore, under applied magnetic fields magnetocrystalline coupling originated by $\mathrm{Tb}$ magnetic arrangement, instead of $\mathrm{Mn}^{3+}$ anisotropy as sometimes suggested in the literature $[37,38]$, is proposed to play a significant role in the polarization flop from $\mathbf{c}$ to $\mathbf{a}$. In other words, the $\mathrm{Tb}$ magnetic order (at $\mathbf{H}_{\mathbf{b}}=5 \mathrm{~T}$.) should induce a motion of the oxygen atoms, which triggers a field-induced transition of the Mn moments into a configuration lying on the $a b$ plane together with the flop of the polarization from the c direction (zero field) to the a direction (high field). To summarize, we show that the contribution from the $\mathrm{Tb}$ magnetic order to the electric polarization in $\mathrm{TbMnO}_{3}$ cannot be ignored.

\section{ACKNOWLEDGMENTS}

The authors are indebted to M. C. Sánchez for the orientation of the single crystals. Financial support from the Spanish MINECO (Projects No. MAT2012-38213-C02-01 and No. MAT2011-23791) and the Diputación General de Aragón (CAMRADS) are acknowledged. We also acknowledge the use of SAI from Universidad de Zaragoza and the Bessy II synchrotron facility for beam time allocation and support during the experiments.
[1] Y. Tokura and S. Seki, Adv. Mater. (Weinheim, Ger.) 22, 1554 (2010).

[2] Y. Tokura, Science 312, 1481 (2006).

[3] M. Fiebig, J. Phys. D: Appl. Phys. 38, R123 (2005).

[4] N. A. Hill, J. Phys. Chem. B 104, 6694 (2000).

[5] D. Khomskii, Phys. 2, 20 (2009).

[6] I. A. Sergienko and E. Dagotto, Phys. Rev. B 73, 094434 (2006).

[7] R. Kajimoto, H. Yoshizawa, H. Shintani, T. Kimura, and Y. Tokura, Phys. Rev. B 70, 012401 (2004).
[8] M. Kenzelmann, A. B. Harris, S. Jonas, C. Broholm, J. Schefer, S. B. Kim, C. L. Zhang, S.-W. Cheong, O. P. Vajk, and J. W. Lynn, Phys. Rev. Lett. 95, 087206 (2005).

[9] J. Voigt, J. Persson, J. W. Kim, G. Bihlmayer, and T. Brückel, Phys. Rev. B 76, 104431 (2007).

[10] N. Aliouane, O. Prokhnenko, R. Feyerherm, M. Mostovoy, J. Strempfer, K. Habicht, K. C. Rule, E. Dudzik, A. U. B. Wolter, A. Maljuk, and D. N. Argyriou, J. Phys.: Condens. Matter 20, 434215 (2008). 
[11] D. Mannix, D. F. McMorrow, R. A. Ewings, A. T. Boothroyd, D. Prabhakaran, Y. Joly, B. Janousova, C. Mazzoli, L. Paolasini, and S. B. Wilkins, Phys. Rev. B 76, 184420 (2007).

[12] V. Cuartero, J. García, G. Subías, J. Herrero-Martín, J. Blasco, and C. R. Natoli, J. Phys.: Conf. Ser. 430, 012101 (2013).

[13] O. Prokhnenko, R. Feyerherm, M. Mostovoy, N. Aliouane, E. Dudzik, A. U. B. Wolter, A. Maljuk, and D. N. Argyriou, Phys. Rev. Lett. 99, 177206 (2007).

[14] S. B. Wilkins, T. R. Forrest, T. A. W. Beale, S. R. Bland, H. C. Walker, D. Mannix, F. Yakhou, D. Prabhakaran, A. T. Boothroyd, J. P. Hill, P. D. Hatton, and D. F. McMorrow, Phys. Rev. Lett. 103, 207602 (2009).

[15] T. Kimura, T. Goto, H. Shintani, K. Ishizka, T. Arima, and Y. Tokura, Nature (London) 426, 55 (2003).

[16] T. Kimura, G. Lawes, T. Goto, Y. Tokura, and A. P. Ramirez, Phys. Rev. B 71, 224425 (2005).

[17] V. Cuartero, J. Blasco, J. A. Rodríguez-Velamazán, J. García, G. Subías, C. Ritter, J. Stankiewicz, and L. Canadillas-Delgado, Phys. Rev. B 86, 104413 (2012).

[18] C.-C. Kao, C. T. Chen, E. D. Johnson, J. B. Hastings, H. J. Lin, G. H. Ho, G. Meigs, J.-M. Brot, S. L Hulbert, Y. U. Idzerda, and C. Vettier, Phys. Rev. B 50, 9599 (1994).

[19] J. Blasco, M. C. Sánchez, J. García, J. Stankiewicz, and J. Herrero-Martín, J. Cryst. Growth 310, 3247 (2008).

[20] B. T. Thole, P. Carra, F. Sette, and G. van der Laan, Phys. Rev. Lett. 68, 1943 (1992).

[21] C. Bordel, S. Pizzini, J. Vogel, K. Mackay, J. Voiron, R. M. Galéra, A. Fontaine, P. Auric, J. B. Goedkoop, and N. B. Brookes, Phys. Rev. B 56, 8149 (1997).

[22] S. Stepanow, J. Honolka, P. Gambardella, L. Vitali, N. Abdurakhmanova, T.-C. Tseng, S. Rauschenbach, S. L. Tait, V. Sessi, S. Klyatskaya, M. Ruben, and K. Kern, J. Am. Chem. Soc. 132, 11900 (2010).
[23] R. Biagi, J. Fernandez-Rodriguez, M. Gonidec, A. Mirone, V. Corradini, F. Moro, V. De Renzi, U. del Pennino, J. C. Cezar, D. B. Amabilino, and J. Veciana, Phys. Rev. B 82, 224406 (2010).

[24] L. Tröger, D. Arvanitis, K. Baberschke, H. Michaelis, U. Grimm, and E. Zschech, Phys. Rev. B 46, 3283 (1992).

[25] E. Stavitski and F. M. F. de Groot, Micron 41, 687 (2010).

[26] P. H. Butler, Point Group Symmetry Applications (Plenum Press, New York and London, 1981).

[27] R. D. Cowan, The Theory of Atomic Structure and Spectra (University of California Press, Berkeley, 1981), p. 365.

[28] P. Carra, B. T. Thole, M. Altarelli, and X. Wang. Phys. Rev. Lett. 70, 694 (1993).

[29] P. Carra, H. König, B. T. Thole, and M. Altarelli, Physica B 192, 182 (1993).

[30] N. Abe, K. Taniguchi, S. Ohtani, T. Takenobu, Y. Iwasa, and T. Arima, Phys. Rev. Lett. 99, 227206 (2007).

[31] J. Mareschal, J. Sivardière, G. F. De Vries, and E. F. Bertaut, J. Appl. Phys. 39, 1364 (1968).

[32] S. Quezel F. Techeou, J. Rossat-Mignod, G. Quezel, and E. Roudaut, Physica B\&C 86-88, 916 (1977).

[33] J. B. Gruber K. L. Nash, R. M. Yow, D. K. Sardar, U. V. Valiev, A. A. Uaokov, and G. W. Burdick, J. Lumin. 128, 1271 (2008).

[34] L. Holmes, R. Sherwood, and L. G. Van Uitert, J. Appl. Phys. 39, 1373 (1968).

[35] M. Sikora, C. Kapusta, D. Zaja, W. Tokarz, K. Attenkofer, P. Fischer, E. Goering, and G. Schütz, J. Alloys Compd. 328, 100 (2001).

[36] E. F. Bertaut, Acta Crystallogr., Sect. A: Cryst. Phys., Diffr., Theor. Gen. Crystallogr. A 24, 217 (1968).

[37] M. Mochizuki and N. Furukawa, Phys. Rev. B 80, 134416 (2009).

[38] H. J. Xiang, S.-H. Wei, M.-H. Whangbo, and J. L. F. Da Silva, Phys. Rev. Lett. 101, 037209 (2008). 